\title{
Loose Stool
}

National Cancer Institute

\section{Source}

National Cancer Institute. Loose Stool. NCI Thesaurus. Code C87128.

Frequent, loose, and watery stools are noted in most infants, even if they are fed formula, until they are six to eight weeks old; after that, the stools become firmer and less frequent. 